Racker, E. (1972) J. Membr. Biol. 10, 221-235.

Robinson, B. H., \& Dalton, L. R. (1980) J. Chem. Phys. 72, 1312-1324.

Saffman, P. G., \& Delbrück, M. (1975) Proc. Natl. Acad. Sci. U.S.A. 72, 3111-3113.

Silvius, J. R., \& McElhaney, R. N. (1981) J. Theor. Biol. 88, 135-152.

Smith, L. (1955) Methods Biochem. Anal. 2, 427-434.

Swanson, M. S., Quintanilha, A. T., \& Thomas, D. D. (1980)

J. Biol. Chem. 255, 7494-7502.
Thilo, L., Träuble, H., \& Overath, P. (1977) Biochemistry 16, 1283-1289.

Thomas, D. D., Dalton, L. R., \& Hyde, J. S. (1976) J. Chem. Phys. 65, 3006-3024.

Vik, S. B., \& Capaldi, R. A. (1977) Biochemistry 16, 5755-5759.

Watts, A., Marsh, D., \& Knowles, P. F. (1978) Biochem. Biophys. Res. Commun. 81, 403-409.

Yoshida, S., Orii, Y., Kawato, S., \& Ikegami, A. (1979) J. Biochem. 86, 1443-1450.

\title{
Polymorphic Phase Behavior of Lysopalmitoylphosphatidylcholine in Poly(ethylene glycol)-Water Mixtures ${ }^{\dagger}$
}

\author{
Martin D. King ${ }^{\ddagger}$ and Derek Marsh* \\ Max-Planck-Institut für biophysikalische Chemie, Abteilung Spektroskopie, D-3400 Göttingen, Federal Republic of Germany \\ Received November 28, 1988; Revised Manuscript Received March 17, 1989
}

\begin{abstract}
The polymorphic phase behavior of 1-palmitoyl-2-lyso-sn-glycero-3-phosphocholine dispersions in excess water has been studied as a function of temperature and poly(ethylene glycol) (PEG) concentration, using proton dipolar-decoupled ${ }^{31} \mathrm{P}$ NMR spectroscopy and turbidity measurements. The phase behavior was found to depend on both lipid concentration and PEG concentration, and most of the NMR experiments were conducted at a lipid concentration of $15 \mathrm{mg} / \mathrm{mL}$. At low PEG concentrations (0-12 wt \%), a thermotropic transition occurs at $3-5^{\circ} \mathrm{C}$ with increasing temperature, from an interdigitated lamellar gel $\left(\mathrm{L}_{\beta}{ }^{\mathrm{i}}\right)$ phase to a normal micellar phase. At intermediate PEG concentrations $(12-20 \mathrm{wt} \%)$, thermotropic transitions take place with increasing temperature, first from the lamellar gel phase to a fluid cubic $\left(Q_{\alpha}\right)$ phase and then at higher temperatures from the cubic phase to the micellar phase. At intermediate PEG concentrations above the former range (20-30 wt \%), thermotropic transitions take place with increasing temperature, first from the lamellar gel phase to the cubic phase, then from the cubic phase to a normal hexagonal $\left(\mathrm{H}_{\mathrm{I}}\right)$ phase, and finally from the hexagonal phase to the micellar phase. At high PEG concentrations $(>30 \mathrm{wt} \%)$, a thermotropic transition takes place with increasing temperature from the lamellar gel phase directly to the fluid hexagonal phase. At these high PEG concentrations, the micellar phase is not attained within the accessible temperature range $\left(\leq 90^{\circ} \mathrm{C}\right)$. The kinetics of some of the phase changes are slow with half-times of the order of $15 \mathrm{~min}$, and considerable hysteresis, with coexisting phases, is observed on the downward temperature scans. The turbidity measurements indicate considerable changes in optical density in single phase regions, presumably corresponding to changes in aggregate size. Discontinuities in the temperature dependence of the optical density are observed corresponding to some of the phase boundaries, and conversion to the micellar phase is evidenced by the transition to optical clarity. A pseudo-binary-phase diagram has been constructed as a function of PEG concentration, and comparison with the phase diagram of lysopalmitoylphosphatidylcholine as a function of water content [Arvidson et al. (1985) Eur. J. Biochem. 152, 753-759] indicates that PEG controls the polymorphic phase behavior by reducing water activity. These results are discussed within the context of PEG-induced cell fusion.
\end{abstract}

$\mathbf{P}$ lene glycol) (PEG) ${ }^{1}$ is known to have many in teresting effects in biological systems, among these being the induction of cell fusion (Ahkong et al., 1975; Lucy, 1984). One of the primary effects by which fusion is induced is thought to be the reduction of water activity by PEG, leading to an effective membrane dehydration (Arnold et al., 1983; MacDonald, 1985). A possible mechanism by which this may take place is the modulation of the lipid phase behavior resulting from the reduction in lipid hydration. A reduction of the water content is known to favor formation of the inverted hexagonal phase in lamellar phosphatidylethanolamines (Seddon et al.,

\footnotetext{
This work has been supported in part by Grant Ma 756/2-3 from the Deutsche Forschungsgemeinschaft.

'Present address: Mass Spectrometry and Computer Unit, Chemical Pathology, Queen Charlotte's Maternity Hospital, Goldhawk Rd., London W6 OXG, U.K.
}

1984) and various natural lipid systems (Luzzati, 1968). Thus, it is possible that PEG may induce the formation of inverted phases which promote the nonlamellar lipid topology necessary for fusion. On the one hand, PEG has been shown to induce nonlamellar defects in egg phosphatidylcholine bilayers (Boni et al., 1981) and, on the other hand, to increase the phase transition temperature of dipalmitoylphosphatidylcholine (Tilcock \& Fisher, 1979). The latter is consistent with the effects of decreasing water activity, since in this system the lipid remains in a lamellar phase down to low water contents.

A partial phase diagram has recently been published for the palmitoyllysophosphatidylcholine $\left(\mathrm{C}_{16}\right.$ lyso $\left.\mathrm{PC}\right)$ /water system (Arvidson et al., 1985). At high water contents and tem-

\footnotetext{
${ }^{1}$ Abbreviations: $\mathrm{C}_{16}$ lysoPC, 1-palmitoyl-2-lyso-sn-glycero-3phosphocholine; PEG, poly(ethylene glycol); NMR, nuclear magnetic resonance.
} 
peratures between 20 and $50^{\circ} \mathrm{C}$, the lipid molecules are in a micellar state, and with decreasing water content, the micelles aggregate to form a cubic phase of novel structure (Eriksson et al., 1985). At yet lower water content, a further transition takes place to a normal hexagonal phase consisting of lipid cylinders in water. For the system in excess water, it has also been demonstrated that a transition from the micellar phase to an interdigitated lamellar gel phase takes place on incubation at low temperature (Wu et al., 1981; Huang et al., 1981).

In the present work, we have investigated the phase behavior of $\mathrm{C}_{16}$ lysoPC as a function of temperature and of PEG concentration in the suspending aqueous phase. The purpose of this work was to determine whether the various nonlamellar phases mentioned above could be induced by increasing PEG concentration and to determine the phase boundaries between the different nonlamellar and lamellar phases. Comparison with the phase diagram of $\mathrm{C}_{16}$ lysoPC as a function of water content indicates that PEG can control lipid polymorphism by reducing water activity. It is felt that the principles established by this study could well have a direct relevance for the mechanism of PEG-induced cell fusion.

\section{Materials AND METHODS}

Poly (ethylene glycol) (PEG; reinst, mp 60-66 ${ }^{\circ} \mathrm{C}$ ) of average molecular weight $6000-7500$ was obtained from Baker Chemicals (Deventer, Netherlands), and palmitoyllysophosphatidylcholine $\left(\mathrm{C}_{16}\right.$ lysoPC; $99 \%$ purity) was from Sigma (St. Louis, MO).

Samples for ${ }^{31} \mathrm{P}$ NMR were prepared by dissolving $30 \mathrm{mg}$ of $\mathrm{C}_{16}$ lysoPC in $\mathrm{CH}_{2} \mathrm{Cl}_{2} / \mathrm{CH}_{3} \mathrm{OH}(2: 1 \mathrm{v} / \mathrm{v})$, evaporating in a stream of dry $\mathrm{N}_{2}$, and removing residual solvent under vacuum overnight. The dry lipid was then dispersed at room temperature in $2 \mathrm{~mL}$ of the required $\mathrm{PEG}$ solution in water (pH 5) and transferred to a 10-mm stoppered NMR tube. At the lowest PEG concentrations, the samples were slow to attain the gel phase and were incubated in the freezer for several days. PEG concentration is expressed as weight percent, i.e., 100[weight of PEG/(weight of PEG + water)]. Analysis by thin-layer chromatography (using the solvent systems $\mathrm{CH}_{2} \mathrm{Cl}_{2} / \mathrm{MeOH} / 30 \%$ ammonia, $65: 35: 5 \mathrm{v} / \mathrm{v}$, and hexane/ diethyl ether, $1: 1 \mathrm{v} / \mathrm{v}$ ) of $\mathrm{C}_{16}$ lysoPC samples in PEG solutions of various concentrations, which were incubated at elevated temperatures for longer times than the NMR measurements were performed, failed to reveal any lipid degradation. In addition, the ${ }^{31} \mathrm{P}$ NMR spectra recorded after temperature cycling were closely similar to those obtained before temperature cycling (cf. Figure 3).

Proton dipolar-decoupled 109- $\mathrm{MHz}^{31 \mathrm{P}}$ NMR spectra were recorded with a Bruker WH-270 spectrometer with a $200-\mathrm{W}$ transmitter, operating in the Fourier-transform mode. The direct free induction decay was collected with an $11-\mu \mathrm{s} 90^{\circ}$ pulse and phase cycling. Use of the Hahn spin-echo sequence did not give rise to visible improvement in the spectral line shapes of the powder patterns. The decoupling power was approximately $20 \mathrm{~W}$, and the duty cycle of the gated decoupling was $0.2 \%$ ( 5 -s recycle delay). Effective chemical shift anisotropies were determined between the points of maximum slope in the outer regions of the powder pattern. For isotropic peaks, the width at half-height was measured.

\section{RESULTS}

Typical proton dipolar-decoupled ${ }^{31} \mathrm{P}$ NMR spectra of $\mathrm{C}_{16}$ lysoPC dispersed in a $23 \mathrm{wt} \%$ aqueous solution of PEG are given in Figure 1. At this PEG concentration, all the various polymorphic phases displayed by $\mathrm{C}_{16}$ lysoPC dispersions

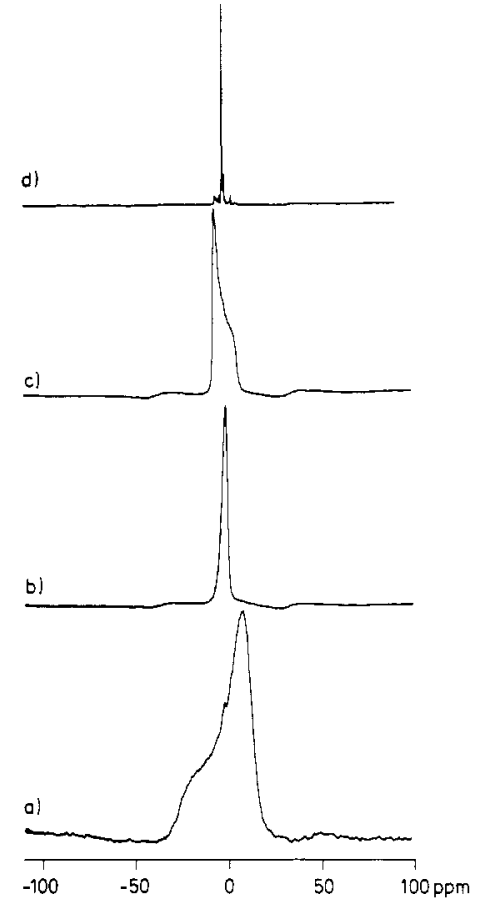

FIGURE 1: Proton dipolar-decoupled 109-MHz ${ }^{31} \mathrm{P}$ NMR spectra of 1-palmitoyl-2-lyso-sn-glycero-3-phosphocholine in $23 \mathrm{wt} \%$ aqueous PEG $\left(M_{\mathrm{r}} 6000-7500\right)$ dispersions. (a) Spectrum recorded at $5{ }^{\circ} \mathrm{C}$; (b) at $12^{\circ} \mathrm{C}$; (c) at $40^{\circ} \mathrm{C}$; and (d) at $80^{\circ} \mathrm{C}$.

can be observed within the experimentally accessible temperature range. At low temperature (Figure 1a), the axially symmetric ${ }^{31} \mathrm{P}$ powder pattern has a negative chemical shift anisotropy, which is characteristic of a lamellar arrangement of the lipid molecules, and the large line widths indicate that the lipids are in a gel phase. At a somewhat higher temperature (Figure $1 \mathrm{~b}$ ), the spectrum changes to a single nearisotropic peak. The nonvanishing width of this latter peak suggests that this is a fluid liquid-crystalline phase, probably with cubic symmetry. Careful examination of the spectral line shape on an expanded horizontal display reveals a shoulder on the low-field side similar to that of the axial powder pattern in the lamellar phase (data not shown). This small anisotropy is most pronounced at low temperatures and is exactly the same as that found in the cubic phase of $46 \mathrm{wt} \% \mathrm{C}_{16}$ lysoPC in water at $25^{\circ} \mathrm{C}$ (Eriksson et al., 1985). Increasing the temperature further (Figure $1 \mathrm{c}$ ) gives rise to an axially symmetric powder pattern with positive chemical anisotropy, which is characteristic of a fluid phase with cylindrical symmetry. Consideration of the shape of the $\mathrm{C}_{16}$ lysoPC molecule suggests that this latter is a normal hexagonal phase. On going to high temperature (Figure 1d), the powder pattern then transforms into a sharp isotropic spectrum characteristic of a micellar dispersion of the lipid. At this point, the sample has changed from a turbid dispersion to a clear solution.

The kinetics of some of the phase changes are slow and involve considerable hysteresis. The time dependence of the change in the relative proportions of the two coexisting phases in $23 \%$ PEG, as recorded by the ${ }^{31} \mathrm{P}$ NMR spectra, is given in Figure 2. Temperatures were chosen such that transitions between the lamellar and cubic phases (Figure 2a), between the cubic and hexagonal phases (Figure $2 b$ ), and between the hexagonal and micellar phases (Figure $2 \mathrm{c}$ ) could be studied. Because of the inate width of the transitions, it is not possible to choose a single temperature at which a complete conversion from one phase to the other takes place. Additionally, because of the hysteresis, it is difficult to define kinetically a unique starting state. The $t=0$ point in Figure 2 is defined as the 




FIGURE 2: Time dependence of the transitions between the various phases of $\mathrm{C}_{16}$ lysoPC in a $23 \mathrm{wt} \%$ PEG aqueous dispersion, deduced from the two-component ${ }^{31} \mathrm{P}$ NMR spectra. (a) Transition from lamellar to cubic phase at $8^{\circ} \mathrm{C}$; (b) transition from cubic to hexagonal phase at $30^{\circ} \mathrm{C}$; (c) transition from hexagonal to micellar phase at $72^{\circ} \mathrm{C}$. The relative proportions of the two phases were determined from the areas under the two spectral components, with the exception of (c), where the ratio of the principal spectral peak heights is used. The final time point in (c) was recorded at $167 \mathrm{~min}$.

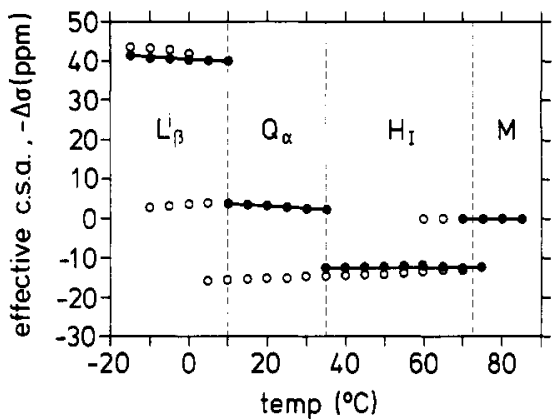

FIGURE 3: Temperature dependence of the effective chemical shift anisotropy, $\Delta \sigma$, of $\mathrm{C}_{16}$ lysoPC in a $23 \mathrm{wt} \%$ PEG aqueous dispersion. Closed circles refer to increasing temperature and open circles to decreasing temperature. Phase identifications refer to the heating curve given by the solid line. Duplicate data points indicate coexisting phases. $\mathrm{L}_{\beta}{ }^{\mathrm{i}}$, interdigitated lamellar gel phase; $\mathrm{M}$, micellar phase; $\mathrm{Q}_{\alpha}$, cubic phase; $\mathrm{H}_{\mathrm{I}}$, normal hexagonal phase.

point immediately at which the desired temperature was achieved. Under the conditions used, it is found that the lamellar to cubic conversion takes place with a half-time of approximately $13 \mathrm{~min}$ and on the order of $100 \mathrm{~min}$ is required to reach close to equilibrium. For the cubic to hexagonal conversion, the half-time is approximately $15 \mathrm{~min}$, and again on the order of $100 \mathrm{~min}$ is required to reach near-equilibrium. The hexagonal to micellar conversion is clearly biphasic, with the fast phase being almost complete in approximately $3 \mathrm{~min}$ and the slow phase taking on the order of $160 \mathrm{~min}$. Since the total acquisition time for a particular spectrum was a minimum of $5 \mathrm{~min}$, the faster rates can only be considered as being qualitative.

The temperature dependence of the effective chemical shift anisotropy in the ${ }^{31} \mathrm{P}$ NMR spectra of $\mathrm{C}_{16}$ lysoPC in $23 \%$ PEG is given in Figure 3. Well-defined transitions are observed between the different phases in the upward temperature scan, whereas hysteresis with considerable temperature ranges of coexisting phases is observed in the downward temperature scan. The chemical shift anisotropy of $-40 \mathrm{ppm}$ observed for the lamellar gel phase in $23 \mathrm{wt} \%$ PEG is very similar to that



FIGURE 4: Temperature-composition phase diagram for $\mathrm{C}_{16}$ lysoPC dispersed in excess aqueous PEG as a function of PEG concentration. Phase boundaries were established from the temperature dependence of the ${ }^{31} \mathrm{P}$ NMR spectra.

observed previously for the lamellar gel phase of $\mathrm{C}_{16}$ lysoPC in buffer alone (van Echteld et al., 1981) and similarly for 1 -stearoyllysophosphatidycholine (Wu et al., 1982), when measured from the published spectra using the conventions detailed under Materials and Methods. The effective chemical shift anisotropy in the cubic phase of -3 ppm at $23 \mathrm{wt} \%$ PEG is very similar to that found in the cubic phase of $46 \mathrm{wt} \%$ $\mathrm{C}_{16}$ lysoPC in water at $25^{\circ} \mathrm{C}$ (Eriksson et al., 1985). The chemical shift anisotropy of $+15 \mathrm{ppm}$ in the hexagonal phase with $23 \mathrm{wt} \%$ PEG at $27^{\circ} \mathrm{C}$ is very similar to that found for the hexagonal phase of $48.8 \mathrm{wt} \% \mathrm{C}_{16}$ lysoPC in water at the same temperature (Arvidson et al., 1985). At higher temperatures, the chemical shift anisotropy is smaller (ca. +12.5 $\mathrm{ppm}$ ), presumably corresponding to the increased degree of molecular motion. The equivalent chemical shift anisotropy in a lamellar phase would be $-25 \mathrm{ppm}$, which is considerably smaller than the -40 to $-45 \mathrm{ppm}$ normally found for diacyl phospholipids in the $\mathrm{L}_{\alpha}$ phase (Seelig, 1978). Even smaller chemical shift anisotropies have been found, however, in the lamellar phases of lysocardiolipins that were attributed to a different conformation in the region of the phosphate group (Powell \& Marsh, 1985). Additionally, effective chemical shift anisotropies in the region of $-12 \mathrm{ppm}$ have been found in the lamellar phases of unsaturated lysophosphatidylethanolamines (Tilcock et al., 1986). In the micellar phase, the chemical shift anisotropy is zero, corresponding to small micelles as expected.

Temperature scans with increasing temperature, of the type shown in Figure 3, have been used to construct a pseudo-binary-phase diagram as a function of PEG content in the dispersing phase. Sufficient time was allowed between temperature measurements such that the ${ }^{31} \mathrm{P}$ NMR spectra were characteristic of the majority equilibrium phase. The phase diagram is given in Figure 4 and displays well-defined regions of stability of the different phases. The low-temperature phase is always a lamellar gel phase, and within the experimentally accessible temperature range for the upper phase boundary, the high-temperature phase is micellar. For PEG concentrations up to $10 \mathrm{wt} \%$, chain melting of the gel phase yields the micellar phase, and for PEG concentrations greater than $30 \mathrm{wt} \%$, the hexagonal phase is obtained on chain melting. The cubic phase is obtained only over a restricted range of temperature and PEG concentrations.

Trial ${ }^{31} P$ NMR experiments also indicated that the phase behavior was dependent on the lipid concentration. It was found that increasing the concentration of $\mathrm{C}_{16}$ lysoPC correlated with changes induced by increasing the PEG concentration. For instance, at a concentration of $100 \mathrm{mg} / \mathrm{mL}$ $\mathrm{C}_{16}$ lysoPC in $20 \mathrm{wt} \%$ PEG, the thermotropic phase behavior was very similar to that at $15 \mathrm{mg} / \mathrm{mL} \mathrm{C}_{16}$ lysoPC in $26 \mathrm{wt} \%$ PEG. These concentration-dependent effects are, of course, 
accentuated by the extremely steep phase boundaries in the region of 15-25 wt \% PEG. Opposite effects were found on decreasing the $\mathrm{C}_{16}$ lyso $\mathrm{PC}$ concentration in this region of the PEG-dependent phase diagram. It was observed that 0.5 $\mathrm{mg} / \mathrm{mL}$ dispersions of $\mathrm{C}_{16}$ lysoPC remained optically clear at all temperatures above $5^{\circ} \mathrm{C}$ in $15 \mathrm{wt} \%$ PEG (cf. Figure 4) whereas turbid, nonmicellar suspensions were obtained at the same $\mathrm{C}_{16}$ lysoPC concentration in $17.5 \mathrm{wt} \%$ PEG (data not shown). This is consistent with a shift in the phase boundary to higher PEG concentrations on reducing the concentration of $\mathrm{C}_{16}$ lysoPC.

\section{DISCUSSION}

The present work demonstrates that a wide range of polymorphic phase behavior of $\mathrm{C}_{16}$ lysoPC can be induced by PEG. Reference to the pseudo-binary-phase diagram in Figure 4 shows that isothermal transitions between the micellar, cubic, and hexagonal phases, or between the micellar, cubic, and lamellar phases, can be induced simply by varying the PEG concentration at the appropriate temperature. Furthermore, the extremely steep phase boundaries found in Figure 4 mean that the phases can be modulated extremely sensitively by only very small changes in PEG concentration in the appropriate range and that these phases will then be stable to quite wide changes in temperature.

The origin of the PEG-induced phase changes lies almost certainly in the well-known ability of PEG to reduce water activity, because of its own propensity to interact with water. This is seen partly from the equivalent effects of increasing PEG concentration and increasing lipid concentration mentioned under Results, and even more clearly from a comparison of Figure 4 with the phase diagram of $C_{16}$ lysoPC in water. A temperature-composition phase diagram for the fluid phases of $\mathrm{C}_{16}$ lysoPC has been determined by Arvidson et al. (1985), by using $\mathrm{X}$-ray diffraction and NMR. At $25^{\circ} \mathrm{C}$, a micellar phase is obtained for $\mathrm{C}_{16}$ lysoPC concentrations up to $37 \mathrm{wt}$ $\%$. A cubic phase is obtained at concentrations between 39 and $46 \mathrm{wt} \% \mathrm{C}_{16}$ lysoPC, and above this concentration, a hexagonal phase is obtained. The phase boundaries of the intermediate cubic phase are very steep, with the phase existing only for temperatures up to approximately $50-60^{\circ} \mathrm{C}$. Above this temperature, a direct transition between the hexagonal and micellar phase is obtained. This phase behavior is clearly isomorphous with that depicted in Figure 4, indicating that the effect of increasing PEG concentration is to move the system through the different regions of the lipid-water phase diagram in the direction of increasing lipid concentration.

The gel-to-fluid region of the lipid-water phase diagram of $\mathrm{C}_{16}$ lysoPC has not been investigated so far. However, the behavior in Figure 4 is very analogous to that observed previously for lysophosphatidylcholines in excess water (Wu et al., 1982; Huang et al., 1982). The transition temperature of $3{ }^{\circ} \mathrm{C}$ obtained in the absence of PEG by ${ }^{31} \mathrm{P}$ NMR is in reasonable agreement with values obtained previously by differential scanning calorimetry and Raman spectroscopy (van Echteld et al., 1980; Huang et al., 1982). An interesting feature of the systems in the absence of PEG is the metastability of the micellar phase at low temperature. Prolonged incubations are required to obtain reversion to the lamellar gel phase on cooling (Wu et al., 1982, 1983). In the presence of PEG, however, it is found that this metastability becomes less pronounced and reversion to the gel phase is more rapid (cf. Figure 3).

The phase identifications given in Figures 3 and 4, on the basis of the ${ }^{31} \mathrm{P}$ NMR spectra, are substantiated by comparison with the phase diagram of $\mathrm{C}_{16}$ lyso $\mathrm{PC}$-water assigned by $\mathrm{X}$-ray diffraction (Arvidson et al., 1985), and with the electron microscopy and Raman spectroscopy of lysophosphatidylcholines in the gel phase (Wu et al., 1985). In particular, the Raman results strongly suggest an interdigitated gel phase $\left(\mathrm{L}_{\beta}{ }^{\mathrm{i}}\right)$, as is found for the crystal structure of deoxylysophosphatidylcholine (Hauser et al., 1980). An interdigitated gel phase has also been demonstrated directly by X-ray diffraction for hydrated stearoyllysophosphatidycholine (Mattai \& Shipley, 1986). Of the fluid phases, the cubic phase is particularly interesting. Both the position of occurrence in the phase diagram (between the micellar and hexagonal phases, when considering isothermal transitions) and the similarity in shape of the ${ }^{31} \mathrm{P}$ NMR spectra suggest that the cubic phase found in PEG solutions is identical with that found in pure water at lipid concentrations of 39-46 wt \%. The latter has been identified with the novel structure suggested by Fontell et al. (1985) for some of the cubic lipid-water systems with space group Pm3n (Arvidson et al., 1985). This structure is composed of units of eight rodlike aggregates with axial ratio around 2 , hence giving rise to a small anisotropy in the ${ }^{31} \mathrm{P}$ NMR spectra (Eriksson et al., 1985). ${ }^{2}$ The ${ }^{31} \mathrm{P}$ NMR spectra indicate that the phase obtained at high PEG concentrations is one with cylindrical symmetry, and comparison with the $\mathrm{X}$-ray data shows that the cylinders are arranged in a hexagonal array. Considerations of the position in the phase diagram and of the shape of the $\mathrm{C}_{16}$ lysoPC molecule show that this phase is of the "oil in water" type, i.e., the normal hexagonal $\left(\mathrm{H}_{\mathrm{I}}\right)$ phase.

The present results could have considerable relevance to the PEG-induced cell fusion technique commonly used in monoclonal antibody production. Clearly, PEG is capable of inducing the nonlamellar topology required for membrane fusion in those lipids for which the appropriate phase boundaries are within an accessible range. The occurrence of steep, almost vertical, phase boundaries such as those in Figure 4 will clearly enhance sensitivity in the corresponding concentration ranges and widen the temperature range over which such effects can be observed. In this connection, it is interesting to note that the standard cell fusion protocol involves suspension in PEG solutions of a concentration close to the maximum used in this study, followed by subsequent dilution to a very low concentration. For lipid systems with phase diagrams similar to that in Figure 4, this would involve the establishment of a different phase with a propensity for membrane-membrane interaction and fusion at high PEG concentration, followed by rearrangement to the original (but fused) membrane topology on retraversing the phase boundaries during subsequent dilution. Clearly, lysophosphatidylcholine alone does not form the phases needed for fluid membrane formation, but the sensitivity of the phase diagram to PEG concentration, demonstrated in Figure 4, establishes the necessary principles. It will be interesting to see in what way PEG affects the phase stability of other lipids undergoing nonlamellar phase transitions, particularly those involving inverted phases compatible with the topology of membrane fusion.

Registry No. C $\mathrm{C}_{16}$ lysoPC, 17364-16-8; PEG, 25322-68-3; $\mathrm{H}_{2} \mathrm{O}$, 7732-18-5.

\section{REFERENCES}

Ahkong, Q. F., Fisher, D., Tampion, W., \& Lucy, J. A. (1975) Nature 253, 194-195.

2 Very recently, an alternative structure has been proposed for the cubic phase with space group $223(\mathrm{Pm} 3 n)$ of the International Tables (Mariani et al., 1988). 
Arnold, K., Pratsch, L., \& Gawrisch, K. (1983) Biochim. Biophys. Acta 728, 121-128.

Arvidson, G., Brentel, I., Khan, A., Lindblom, G., \& Fontell, K. (1985) Eur. J. Biochem. 152, 753-759.

Boni, L. T., Stewart, T. P., Alderfer, J. L., \& Hui, S. W. (1981) J. Membr. Biol. 62, 71-77.

Eriksson, P.-O., Lindblom, G., \& Arvidson, G. (1985) J. Phys. Chem. 89, 1050-1053.

Fontell, K., Fox, K., \& Hansson, E. (1985) Mol. Cryst. Liq. Cryst., Lett. Sect. 1, 9-17.

Hauser, H., Pascher, I., \& Sundell, S. (1980) J. Mol. Biol. 137, 249-264.

Huang, C.-H., Lapides, J. R., \& Levin, I. W. (1982) J. Am. Chem. Soc. 104, 5926-5930.

Lucy, J. A. (1984) Ciba Found. Symp. 103, 28-39.

Luzzati, V. (1968) in Biological Membranes (Chapman, D., Ed.) Vol. 1, pp 71-123, Academic Press, London.

MacDonald, R. I. (1985) Biochemistry 24, 4058-4066.

Mariani, P., Luzzati, V., \& Delacroix, H. (1988) J. Mol. Biol. 204, 165-189.
Mattai, J., \& Shipley, G. G. (1986) Biochim. Biophys. Acta 859, 257-265.

Powell, G. L., \& Marsh, D. (1985) Biochemistry 24, 2902-2908.

Seddon, J. M., Cevc, G., Kaye, R. D., \& Marsh, D. (1984) Biochemistry 23, 2634-2644.

Seelig, J. (1978) Biochim. Biophys. Acta 515, 105-140.

Tilcock, C. P. S., \& Fisher, D. (1979) Biochim. Biophys. Acta $557,53-61$.

Tilcock, C. P. S., Cullis, P. R., Hope, M. J., \& Gruner, S. M. (1986) Biochemistry 25, 816-822.

van Echteld, C. J. A., de Kruijff, B., \& de Gier, J. (1980) Biochim. Biophys. Acta 595, 71-81.

van Echteld, C. J. A., de Kruijff, B., Mandersloot, J. G., \& de Gier, J. (1981) Biochim. Biophys. Acta 649, 211-220.

Wu, W.-G., \& Huang, C.-H. (1983) Biochemistry 22, 5068-5073.

Wu, W.-G., Huang, C.-H., Conley, T. G., Martin, R. B., \& Levin, I. W. (1982) Biochemistry 21, 5957-5961.

\title{
NMR Studies of Exocyclic 1, $N^{2}$-Propanodeoxyguanosine Adducts (X) opposite Purines in DNA Duplexes: Protonated X(syn).A(anti) Pairing (Acidic pH) and $\mathrm{X}($ syn $) \cdot \mathrm{G}($ anti) Pairing (Neutral $\mathrm{pH})$ at the Lesion Site $^{\dagger}$
}

\author{
Michael Kouchakdjian, ${ }^{\ddagger}$ Edmund Marinelli, ${ }^{\S}$ Xiaolian Gao, ${ }^{\ddagger}$ Francis Johnson,,${ }^{\S}$ Arthur Grollman, ${ }^{*, \S}$ and \\ Dinshaw Patel ${ }^{*}, \ddagger$ \\ Department of Biochemistry and Molecular Biophysics, College of Physicians and Surgeons, Columbia University, New York, \\ New York 10032, and Department of Pharmacological Sciences, State University of New York at Stony Brook, Stony Brook, \\ New York 11794 \\ Received October 31, 1988; Revised Manuscript Received March 6, 1989
}

\begin{abstract}
Proton and phosphorus two-dimensional NMR studies are reported for the complementary d(C1-A2-T3-G4-X5-G6-T7-A8-C9)-d(G10-T11-A12-C13-A14-C15-A16-T17-G18) nonanucleotide duplex (designated X-A 9-mer) that contains a 1, $N^{2}$-propanodeoxyguanosine exocyclic adduct, X5, opposite deoxyadenosine A14 in the center of the helix. The NMR studies detect a pH-dependent conformational transition; this paper focuses on the structure present at $\mathrm{pH}$ 5.8. The two-dimensional NOESY studies of the X.A 9-mer duplex in $\mathrm{H}_{2} \mathrm{O}$ and $\mathrm{D}_{2} \mathrm{O}$ solution establish that X5 adopts a syn orientation while A14 adopts an anti orientation about the glycosidic bond at the lesion site. The large downfield shift of the amino protons of $\mathrm{A} 14$ demonstrates protonation of the deoxyadenosine base at $\mathrm{pH} 5.8$ such that the protonated $\mathrm{X} 5$ (syn) $\cdot \mathrm{A} 14$ (anti) pair is stabilized by two hydrogen bonds at low $\mathrm{pH}$. At $\mathrm{pH} 5.8$, the observed NOE between the $\mathrm{H} 8$ proton of $\mathrm{X} 5$ and the $\mathrm{H} 2$ proton of $\mathrm{A} 14$ in the X-A 9-mer duplex demonstrates unequivocally the formation of the protonated X5(syn) $\cdot \mathrm{A} 14$ (anti) pair. The 1, $N^{2}$-propano bridge of X5(syn) is located in the major groove. Selective NOEs from the exocyclic methylene protons of $\mathrm{X} 5$ to the major groove $\mathrm{H} 8$ proton of flanking G4 but not G6 of the G4-X5-G6 segment provide additional structural constraints on the local conformation at the lesion site. A perturbation in the phosphodiester backbone is detected at the C13-A14 phosphorus located at the lesion site by ${ }^{31} \mathrm{P}$ NMR spectroscopy. The two-dimensional NMR studies have been extended to the related complementary X.G 9-mer duplex that contains a central X5.G14 lesion in a sequence that is otherwise identical with the X.A 9-mer duplex. The NMR experimental parameters are consistent with formation of a pH-independent X5(syn).G14(anti) pair stabilized by two hydrogen bonds with the $1, N^{2}$-propano exocyclic adduct of $\mathrm{X} 5(\mathrm{syn})$ located in the major groove.
\end{abstract}

$\mathbf{S}$

\footnotetext{
${ }^{\dagger}$ This research was supported by a Columbia University Faculty Start-Up Grant to D.J.P. and NIH Grants ES-04068 and CA-17395 to A.P.G.

'Columbia University.

State University of New York at Stony Brook.
}

acid adducts with DNA [reviewed in Singer and Barsch (1986), Singer and Grunberger (1983), and Basu and Essigmann (1988)]. Cyclic derivatives may affect base pairing, and it has been suggested that these structures play an important role in mutagenesis and carcinogenesis.

Acrolein reacts readily with deoxyguanosine to form three cyclic adducts (Chung et al., 1984). The reaction of this 